\title{
Key role of the polarization anisotropy of water in modeling classical polarizable force fields
}

\author{
Jean-Philip Piquemal ${ }^{1}$,Riccardo Chelli ${ }^{2,3}$, Piero Procacci ${ }^{2,3}$, and Nohad Gresh ${ }^{4}$ \\ ${ }^{1}$ Laboratoire de Chimie Théorique, Université Pierre et Marie Curie (Paris VI), case 137, 4 \\ place Jussieu, 75252 Paris Cedex 05, France.jpp@1ct.jussieu.fr \\ ${ }^{2}$ Dipartimento di Chimica, Università di Firenze, Via della Lastruccia 3, I-50019 Sesto \\ Fiorentino, Italy. \\ ${ }^{3}$ European Laboratory for Non-linear Spectroscopy (LENS), Via Nello Carrara 1, I-50019 \\ Sesto Fiorentino, Italy. \\ ${ }^{4}$ Laboratoire de Pharmacochimie Moléculaire et Cellulaire, U648, INSERM, IFR \\ Biomédicale, 45 rue des Saints-Pères, 75006 Paris, France.
}




\begin{tabular}{|c|c|c|}
\hline & Ab initio & SIBFA \\
& KM (RVS) & $\mathbf{E}_{\text {pol }}\left(\mathbf{E}_{\mathrm{pol}}{ }^{*}\right)$ \\
\hline BC & $-9.8(-8.1)$ & $-9.0(-7.2)$ \\
\hline t-HBC & $-5.5(-4.7)$ & $-3.87(-3.6)$ \\
\hline l-HBC & $-17.3(-14.5)$ & $-18.2(-14.0)$ \\
\hline
\end{tabular}

Polarization energies (in $\mathrm{kcal} / \mathrm{mol}$ ) computed at the HF/CEP 4-31G(2d) level using the Kitaura-Morokuma (KM) and Restricted Variational Space (RVS) approaches compared to SIBFA. $E_{\mathrm{pol}}$ is the fully relaxed polarization energy and Epol* corresponds to a single iteration of the iterative procedure as described in the manuscript. The data refer to the water chains with 12 molecules with oxygen-oxygen distance of $2.97 \AA$ Á. 